\title{
Évaluer les objets de luxe au Moyen Âge
}

\author{
Laurent FELLER \\ Université Paris 1 \\ Panthéon-Sorbonne/IUF \\ feller@univ-paris1.fr
}

\section{RÉSUMÉ}

Les objets de luxe sont des indicateurs de richesse et de rang. Leur possession désigne la place occupée par leurs détenteurs dans une hiérarchie, qu'elle soit sociale, politique ou économique. Ils ont également des fonctions importantes dans l'échange et la mise en circulation des richesses: réserves de valeur, ils peuvent également servir de moyen de paiement à côté ou en plus des monnaies.

Mots clés: Évaluer, Statut, Richesse, Rang, Circulations non marchandes.

\section{Assessing Luxury Objects in the Middle Ages}

\begin{abstract}
Luxuries are indicators of wealth and social rank. Their possession determines the place occupied by their owners in a hierarchy, whether social, political or economic. They also have important roles in the exchange and circulation of wealth. Luxuries, seen as stores of value, may also serve as a means of payment together with or instead of coins.
\end{abstract}

Key words: Assessing, Status, Wealth, Rank, Non-Market Exchange. 
La catégorie des objets de luxe est une subdivision du groupe des objets de propriété et désigne des biens rares du fait de la matière précieuse qu'ils renferment ou du fait de l'importance du travail humain qu'ils incorporent. Leur valeur peut également provenir de leur beauté, à condition toutefois que celle-ci soit liée à la préciosité du matériau et à la qualité du travail effectué sur eux. Ces objets sont, en tout état de cause, des signes de statut. Ils relèvent du superflu que seuls l'aisance, la richesse ou le pouvoir peuvent procurer. La possession d'objets de luxe place leur détenteur à l'intérieur d'une hiérarchie: les apparences sont essentielles au Moyen Âge et la beauté, la rareté et le prix des objets possédés et exhibés, qu'il s'agisse de vêtements ou de bijoux, de chevaux ou d'armes, permet de classer et de situer sur l'échelle de la richesse et du pouvoir.

M. Godelier a, voici quelques années, proposé une typologie des biens de propriété qui est tout à fait efficace pour contribuer à décrire le rapport des hommes aux objets. S'appliquant aux sociétés d'au-delà du «Grand Partage», aux sociétés dites primitives, elle est tout à fait éclairante pour notre période ${ }^{1}$. Il propose en effet de distinguer «les choses que l'on vend, celles que l'on donne, et celles qu'il ne faut ni vendre ni donner mais garder pour transmettre». Les objets de luxe peuvent s'intégrer à l'intérieur de chacune de ces trois catégories mais la notion même de luxe permet d'introduire implicitement ou explicitement la question de la valeur comme élément essentiel de la classification des objets. Les choses que l'on vend et qui, par définition, ont une valeur se différencient des objets précieux qui, pour leur part, circulent selon une logique particulière laquelle n'est pas nécessairement marchande - mais peut l'être aussi. Les objets sacrés, pour leur part, ne doivent en aucune manière circuler sauf par le biais de la transmission héréditaire ou simplement par leur maintien à l'intérieur d'une institution: c'est, par exemple, le statut particulier des objets constituant les trésors d'églises, inaliénables parce que détenus seulement en usufruit par les clercs ${ }^{2}$. Or, les objets de luxe ont ceci de particulier qu'ils peuvent relever en même temps des trois groupes (usage, précieux et sacré). Mettre cette catégorie particulière d'objets en relation avec l'évaluation revient à poser en premier lieu une question technique ou pratique, celle de l'organisation de la circulation des objets à l'intérieur d'économies et de sociétés qui ne connaissent que partiellement le recours au marché et où les transactions mettant en cause des valeurs ne sont pas nécessairement des transactions marchandes: le pretium, le prix, peut très souvent être compris comme synonyme de contrepartie dans un échange sans référence à un marché.

La question de la valeur des choses ne saurait de plus se limiter à la seule question de la mesure monétaire de celle-ci, les objets de luxe se définissant aussi bien par

1 M. GODELIER, Au fondement des sociétés humaines. Ce que nous apprend l'anthropologie, Paris, 2007, p. 67 sv.

2 La question de la propriété des biens d'église est évidemment au cœur de nombreuses réflexions d'historiens et a donné lieu à une bibliographie pléthorique. Voir, en dernier lieu, S. WOOD, The Proprietary Church in the Medieval West, Oxford, 2006, et le très important commentaire critique de J. NELSON, "Church Properties and the Propertied Church: Donors, the Clergy and the Church in Medieval Western Europe from the Fourth Century to the Twelfth", English Historical Review, CXXIV, 507 (2009), pp. 355-374 ; voir également V. TONEATTO, Les banquiers du Seigneur. Évêques et moines face à la richesse (IVE-début du IXe siècle), Rennes, 2012. 
leur fonction que par leur rareté. La possession de certains types d'objets est en effet liée à des fonctions particulières ou à des conduites de prestige, ce qui les place automatiquement, dans des catégories différenciées à l'égard desquelles leurs détenteurs ne se comportent pas de la même manière qu'à l'égard des objets d'usage, et non connotés de rôles symboliques. Il en est ainsi par exemple des armes durant le haut Moyen Âge.

Á côté de celle de leur coût, la question de savoir à quoi ils servent, quel est leur usage et quelle est leur fonction est tout à fait essentielle. Dans la gamme des biens meubles détenus par des individus ou par des familles, les objets de luxe occupent en effet une place particulière. Ce sont d'abord des indicateurs de rang et leur possession marque la position statutaire ou la position sociale de leurs possesseurs. Cela fait que leur circulation obéit à des règles et à des contraintes qui les placent souvent en marge du marché. Ce sont aussi, de façon parfois contradictoire, des réserves de valeur, la thésaurisation s'effectuant sous la forme de bijoux, d'ornements ou d'œuvres d'art. Saisir les raisons et les modalités de la transformation de ces objets en moyens d'échange ou de paiement est l'une des difficultés que rencontre l'histoire économique du Moyen Âge: la disponibilité en numéraire n'épuise pas, en effet, la question des moyens de paiement et tout objet peut ainsi, à tout moment, devenir lui-même vecteur de l'échange sans que sa valeur d'usage en soit affectée.

Réfléchir sur la mesure de la valeur des objets de luxe amène ainsi à poser une série de questions difficiles concernant le rapport entre les hiérarchies sociales et la possession et l'ostentation d'objets précieux, concernant aussi la question de leur usage (comment se sert-on des objets de luxe?) et de leur circulation, par le marché, le don mais aussi le vol et le pillage.

\section{Objets de luxe et hiérarchies}

La détention et l'exhibition de certains types d'objets classe leur possesseur et marque leur appartenance à des groupes. Quelques évidences s'imposent. Par exemple la détention d'armes est le signe de l'appartenance au groupe des guerriers. Les armes cependant ne se valent pas toutes; elles sont porteuses de symboliques particulières et, en particulier, leur qualité indique clairement la place d'un individu non seulement dans un groupe social, mais aussi dans une fratrie. Analysant le testament d'Evrard de Frioul, Cristina La Rocca et Luigi Provero ont ainsi relevé que les épées jouaient un rôle essentiel dans la formalisation de la hiérarchie familiale de la famille des Unroch dans la seconde moitié du IX ${ }^{e}$ siècle. L'aîné, Unroch, doit hériter du pouvoir que détenait son père. Il reçoit la majeure partie des biens fonciers et une épée dorée; les puinés reçoivent pour leur part des épées dont la valeur est liée à leur place dans la succession. Ainsi, Bérenger se voit attribuer deux épées ornées d'or et d'argent, Adalhard deux épées ornées d'or et d'ivoire et le quatrième, Rodolphe, trois épées simples ${ }^{3}$.

3 C. LA ROCCA et L. PROVERO, "The Dead and Their Gifts. The Will of Eberhard, Count of Friuli, and His Wife Gisela, Daughter of Louis the Pious (863-864)", F. THEUWS et J.L. NELSON (éd.), Rituals of Power. From Late Antiquity to the Early Middle Ages, Leiden-Boston-Köln, 2000, pp. 225-280. 
La valeur est liée ici au symbole signifié par la matière utilisée. L'or seul vient en tête, puis l'association or et argent pour Bérenger et or et ivoire pour Adalhard. Le dernier né a, pour sa part, les deux épées et une somme importante versée en mancusi, c'est-à-dire en monnaies d'or arabes. Le rang est désigné par la qualité des matériaux utilisés pour les armes. Leur valeur est en fait incommensurable: l'objet sert essentiellement à déterminer la place de celui qui le reçoit et cette fonction est incompatible avec l'affectation d'une valeur monétaire. En revanche, le legs fait au dernier né, parce qu'il est partiellement monétarisé, pose problème. Rodolphe pourrait avoir reçu une forme de compensation pour le rang qu'il n'a pas et pour les objets qu'on ne lui attribue pas sous la forme d'un trésor en or. Les mancosi, en effet, ne peuvent circuler dans l'empire sans une conversion préalable en deniers, devenue la seule espèce théoriquement disponible et courant dans le public. Concrètement, ils ne peuvent servir qu'à la thésaurisation ou à l'achat de nouvelles parures dans le monde arabo-musulman ${ }^{4}$. Leur valeur économique est indirecte et est liée au prestige qu'ils peuvent éventuellement permettre d'acquérir en surcroît par des achats bien dirigés. Leur valeur particulière vient ici du mode d'acquisition et de mise en circulation : ils font partie d'un legs et ont été désignés par le donateur. Liés à l'échange marchand et non à la transmission familiale, les objets acquis à prix d'argent ont en revanche un statut inférieur à celui que leur confère le don ou le legs.

Dans une société ancienne, cette question de l'équivalent monétaire de l'objet cédé est donc extrêmement difficile à aborder, dans la mesure précisément où ce qui fait la valeur de l'objet destiné à être transmis est d'échapper sinon pour toujours du moins le plus longtemps possible à la circulation par le marché.

Le choix des objets légués établit ainsi des hiérarchies à l'intérieur de la famille. Il permet également à un seigneur de maintenir après sa mort l'ordre qu'il a établi de son vivant entre ses vassaux. Ainsi, par exemple, le comte de Mâcon, Heccard, faisant son testament en 876 , dispose à la fois de ses biens meubles et de certains éléments de son patrimoine foncier en faveur de différents légataires ${ }^{5}$. Les objets tirés du trésor sont utilisés avec beaucoup d'intelligence pour honorer des proches qui les reçoivent comme des signes de distinction, c'est-à-dire comme des gages de leur rang à l'intérieur du cercle des amis et des vassaux du comte. Les bijoux, les livres et les armes sont ainsi mobilisés, comme dans le testament d'Evrard, une mention toute particulière devant être faite de la broigne du comte qui passe à l'un de ses proches. Le testament d'Evrard de Frioul comportait lui aussi mention de nombreuses armes défensives dont la valeur monétaire comme la valeur symbolique était importante : la broigne est en effet caractéristique de l'armement franc et a longtemps été l'un des éléments de la supériorité militaire carolingienne sur le champ de bataille. Heccard d'Autun organise donc la mise en circulation de pièces de son trésor et des objets

4 Sur ces circulations, voir en dernier lieu, E. MANZANO, "Circulation des biens et des richesses entre al-Andalus et l'Occident européen durant les 'siècles obscurs",' L. FELLER et A. RODRÍGUEZ (éd.), Objets sous contrainte. Circulation des objets et valeur des choses au Moyen Âge, Paris, 2013, pp. 148-180.

5 O. BRUAND, "La gestion du patrimoine des élites en Autunois. Le prieuré de Perrecy et ses obligés (fin IX $^{\mathrm{e}}-\mathrm{X}^{\mathrm{e}}$ siècle)", J.-P. DEVROEY, L. FELLER et R. LE JAN (éd.), Les élites et la richesse au haut Moyen Âge (Actes du colloque tenu à Bruxelles les 13-15 mars 2008), Turnhout, 2008, pp. 233-250. 
précieux qu'il peut posséder afin de transmettre, avec les objets, à la fois les insignes de son pouvoir politique et militaire et ceux d'un prestige personnel lié à sa richesse aussi bien qu'à son pouvoir.

La valeur de ce qui est légué ici compte donc moins que la nature de l'objet transmis et que la place hiérarchique ou statutaire de celui qui donne. Les deux éléments joints ensembles contribuent à classer le bénéficiaire du don à l'intérieur d'un groupe. Les pièces qui composent le trésor peuvent ainsi, pour certaines d'entre elles, être données à des fidèles ou à des amis, permettant ainsi à ceux-ci de partager la richesse d'un puissant de plus haut rang par la possession d'objets liés à sa personne ou à sa fonction. Les objets de plus ont manifestement un statut à part dans la composition de la fortune d'Heccard. Alors que les terres léguées doivent être rachetées par les bénéficiaires du legs, les objets sont cédés à titre purement gracieux. Heccard exige en effet un pretium, une contrepartie monétaire, aux legs de terre qu'il fait, et ce pretium est lourd. Les sommes ainsi accumulées par les exécuteurs testamentaires doivent être distribuées aux pauvres et aux amis d'Eccard, aussi bien les plus nobles que les serviteurs. Ainsi, la mort du comte est-elle l'occasion de mettre en circulation une fraction non négligeable de la richesse de son entourage et de la redistribuer partiellement en se servant de ses propres biens comme de leviers. Contraignant les bénéficiaires à payer pour entrer en possession des legs faits en terres, il les contraint en fait à déthésauriser, à remettre en circulation des valeurs immobilisées, qu'il s'agisse de monnaies ou d'objets précieux. Les biens meubles, pour leur part, échappent à cette logique de la mise en circulation. Ils sont donnés à une personne particulière qui doit conserver l'objet ou, s'agissant de chevaux ou d'armes, s'en servir.

Les mêmes objets qui circulent par le legs le font aussi par le don, dont le maniement a des effets sociaux complexes proches cependant de ceux que le legs produit ${ }^{6}$. Mais, alors que le legs d'objets de valeur a pour effet de prendre acte des situations au moment du décès du testateur, le don a des effets dynamiques, puisqu'il permet à la fois d'organiser la circulation des richesses, et d'établir et de consolider des liens entre les hommes en désignant la place de ceux qui reçoivent à l'intérieur d'une hiérarchie.

Le luxe plus ou moins grand de ce qui fait l'objet du don a un sens et la valeur de ce qui circule a un effet immédiat, celui d'établir un classement des individus en leur affectant un rang dans une hiérarchie ${ }^{7}$. Au XI ${ }^{e}$ siècle en Westphalie l'évêque de Paderborn, Meinwerk, a donné une très grande quantité d'objets précieux. Il a obtenu en contrepartie, dans des échanges qui n'ont apparemment aucun caractère commercial, des terres en grand nombre. La partie du patrimoine mobilier cédée par Meinwerk

6 Sur le don et les problématiques qui lui sont liées : E. MAGNANI SOARES-CHRISTEN (éd.), Don et sciences sociales. Théories et pratiques croisées, Dijon, 2007 et, plus spécialement E. MAGNANI SOARESCHRISTEN, "Les médiévistes et le don. Avant et après la théorie maussienne", ibid., p. 15-29.

7 T. REUTER, "Property transactions and social relations between rulers, bishops and nobles in early eleventh-century Saxony : the evidence of the Vita Meinwerci", W. DAVIES et P. FOURACRE (éd.), Property and power in the early middle ages, Cambridge, 1995, pp. 165-199; L. FELLER, "Transformation des objets et valeur des choses. L'exemple de la Vita Meinwerici”, L. FELLER et A. RODRÍGUEZ (éd.), op. cit., 2013, pp. 91-122. 
consistait en armes offensives et défensives, en chevaux, pièces de draps, fourrures, vêtements divers, tuniques, peaux tannées ou non.

Meinwerk donne aussi, à côté de ces objets précieux qui rentrent tous dans la catégorie des objets de luxe, de l'or et de l'argent, le plus souvent sous une forme non monnayée. La contrepartie est fournie immédiatement par les terres dont les aristocrates du diocèse lui font donation à leur tour. Il s'agit en fait d'échanges de dons qui permettent à l'évêque d'accroître son patrimoine foncier et d'établir ou de consolider sa domination sur la société aristocratique de la Saxe occidentale. La procédure permet également aux membres de l'élite sociale d'obtenir des objets de valeur dont ils pouvaient être dépourvus (armes et chevaux) et dont la provenance (les écuries et l'armurerie de l'évêque) pouvait contribuer à accroître leur statut ${ }^{8}$. L'acquisition de ces objets allait de pair avec une augmentation parfois conséquente de leurs revenus, Meinwerk cédant, en plus des objets de luxe, des terres en viager et octroyant également souvent une allocation en nourriture.

La connaissance de la valeur des choses mises en circulation dans le cadre d'échanges non commerciaux permet aux contemporains de donner immédiatement une signification aux circulations des objets effectuée lors des échanges de don. Il ne suffit pas en effet qu'un objet ait appartenu à un grand ou qu'il provienne de ses réserves, de son trésor, pour que le mettre en circulation par un don ou un legs ait un sens. Il faut, aussi qu'il ait une valeur, connue de tous, qu'il soit ainsi estimé en fonction de sa rareté, de sa qualité ou de sa beauté bref, qu'il soit placé à l'intérieur d'une catégorie, celle des objets précieux, et que ce classement soit à la fois spontané, immédiat et consensuel, c'est-à-dire que tous les acteurs soient capables de reconnaître en lui les éléments qui le font appartenir à un groupe plutôt qu'à un autre. C'est à cela que doivent servir les cérémonies au cours desquelles les objets sont donnés ou échangés. Les remises d'armes du haut Moyen Âge ont cette fonction, mais aussi les assemblées au cours desquelles les souverains remettaient les cadeaux qu'ils faisaient à leurs proches ou à leurs amis, aux ambassadeurs aussi, bref à tous ceux que l'on voulait d'une manière ou d'une autre honorer'. Les rituels d'investiture, parce qu'ils se servent d'objets comme vecteurs des pouvoirs ou comme représentation des richesses qui circulent, servent à rendre publics le classement des hommes, l'assignation du rang qui leur est donné au moment où le souverain attribue la chose qui représente le don.

8 F. BOUGARD, D. IOGNA-PRAT et R. LE JAN (éd.), Hiérarchie et stratification sociale dans l'Occident médiéval (400-1100), Turnhout, 2008 (Collection Haut Moyen Age, 6); F. BOUGARD, G. BÜHRERTHIERRY et R. LE JAN, "Les élites du haut Moyen Âge. Identités, stratégies, mobilité", Annales. Histoire, Sciences Sociales, 68, 4 (2013), pp. 1079-1112.

9 R. LE JAN, "Remises d'armes et rituels du pouvoir chez les Francs: continuités et ruptures de l'époque carolingienne”, R. LE JAN (éd.), Femmes, pouvoir et société, Paris, 2001, pp. 171-189 ; J.L. NELSON, "Munera", J.-P. DEVROEY, L. FELLER et R. LE JAN (éd.), Les élites et la richesse au Moyen Âge, Turnhout, 2010, pp. 383-401. 


\section{Lois somptuaires et consommations urbaines}

La société du haut Moyen Âge est une société pauvre, où seuls les membres de la plus haute aristocratie peuvent avoir accès à des objets de luxe. Les choses sont très différentes durant le Moyen Âge central et le bas Moyen Âge, à partir du XIII siècle. Le développement de la production et des échanges ont eu pour conséquence matérielle de mettre à la disposition des élites comme des membres des classes dominées un nombre croissant d'objets. La question du luxe et celle de la possession d'objets de valeur deviennent alors très importantes dans des sociétés urbaines enrichies par les activités de production et d'échange, à la fois pour des questions de prestige et de rang, mais aussi pour des raisons d'ordre éthiques et économiques.

Les consommateurs potentiels sont en nombre croissant. Aux consommateurs traditionnels d'objets de luxe que sont les clercs et les nobles sont venus s'ajouter les marchands et certains artisans et sans doute les plus aisés des paysans. Les bouleversements sociologiques survenus en ville modifient de plus considérablement le rapport aux objets, dont la typologie en même temps, s'étend. On trouve en ville des consommateurs potentiels plus nombreux, et une plus grande offre d'objets de toute nature s'y développe. Les objets luxueux et coûteux, servant à la parure, comme au vêtement, en particulier, sont disponibles en plus grande quantité. Les textes littéraires, lorsqu'ils relèvent de la catégorie des satires, nous montrent bien que, objets de regrets, souvent parce que le locuteur ne peut pas se les offrir, ils sont présents à l'horizon de ses désirs: les listes souvent burlesques des poètes du XIII ${ }^{e}$ siècle nous montrent bien la présence des choses dans la vie et leur importance dans le quoti$\operatorname{dien}^{10}$.

Le contrôle de l'accès à ces objets, comme à certains modes de consommation ostentatoire est en ville un sujet politique de toute première importance à partir du $\mathrm{XIV}^{\mathrm{e}}$ siècle. La crise de la fin du Moyen Âge n'a en effet pas fait chuter de façon significative la consommation de produits de luxe et la demande s'en est toujours maintenue à un niveau élevé ${ }^{11}$. Or, dès l'instant où les objets désirables sont plus nombreux et d'une meilleure qualité et que, dans le même temps, la demande solvable en de tels biens s'accroît, le risque est réel de voir se brouiller les repères sociaux et les distinctions entre les différents groupes constituant la société. Le problème est alors celui de l'extension des usages du luxe à des catégories sociales qui n'y avaient pas accès auparavant. Au cœur du problème se trouve naturellement la place de l'argent dans la vie sociale et les capacités subversives de celui-ci.

L'argent a en effet suffisamment de force pour subvertir les hiérarchies et les apparences qui les désignent ou les maintiennent. Les prédicateurs du XIV siècle le savent

10 Il s'agit très souvent il est vrai de denrées alimentaires et de biens de première nécessité, comme dans le dit de la maille: De la maaille, M. JEAY (éd.), Hyperlistes. La liste comme principe poétique, XIIeXVIe siècles, Hamilton, 2002, http://tapor.mcmaster.ca/ hyperliste/home.htm (visité le 2 mai 2014) (= P. MÉNARD, "Le dit de la maille", Mélanges de langue et de littérature médiévales offerts à Pierre Le Gentil, Paris, 1973, pp. 541-552).

11 M.G. MUZZARELLI et A. CAMPANINI (éd.), Disciplinare il lusso. La legislazione suntuaria in Italia e in Europa tra Medievo ed Età moderno, Rome, 2003 ; M. CARBONI et M.G. MUZZARELLI (éd.), In Pegno. Oggetti in transito tra valore d'uso e valore di scambio (secc. XIII-XX), Bologne, 2012. 
bien, comme le savent aussi les Mendiants qui réfléchissent à la pauvreté ainsi qu'à l'usage pauvre des choses ${ }^{12}$. On a dit tout à l'heure que l'un des soucis des nobles du haut Moyen Âge, outre la création de hiérarchies à l'intérieur des familles ou de la société tout entière, était d'organiser la circulation des richesses: de là la nécessité de payer pour les pauvres une certaine somme d'argent afin d'entrer en possession d'un legs. Ici, ce souci est étendu au comportement de l'ensemble de la classe dominante, avec le même double souci, celui de la stabilité des hiérarchies, celui d'une circulation convenable des richesses.

C. de la Roncière a montré que, dans les années 1360 à Florence, l'élévation des salaires, plus rapide que celle du prix des denrées alimentaires, permit à des artisans d'avoir accès à certains types de produit qui leur étaient interdits auparavant. La qualité de la nourriture, celle du pain et celle du vin s'accrut ${ }^{13}$. Mais, surtout, ils purent améliorer de façon très sensible la qualité de leur vestiaire, acquérant des ornements ou des pièces de vêtement de meilleure qualité, voire considérés comme luxueux. Cela se voit tout d'abord dans la possession de bijoux ou d'accessoires vestimentaires comme les ceintures en soie brodées d'or qui sont parfois accessibles à des hommes et des femmes considérés comme pauvres, parce que ce sont des travailleurs. Or, les sociétés urbaines, particulièrement italiennes, ont développé, à l'égard du vêtement, une attitude de plus en plus crispée montrant l'existence d'une peur de voir se confondre les genres, les fonctions et les statuts. Les règlements somptuaires sont là pour s'efforcer de porter remède à ce qui apparaît comme un désordre majeur, de nature à provoquer la colère divine. L'apparence des hommes, réglée par les ordonnances ou les lois somptuaires, est le signe de l'harmonie qui doit régner dans la société ${ }^{14}$.

L'argent est susceptible de faire en sorte que les conditions soient indiscernables et que les marchands, les artisans ne soient plus distingués des nobles par leur vêtement. Les règlements s'attachent ainsi non seulement à limiter le coût des vêtements, mais aussi à définir les matières et les quantités qu'il est licite d'employer pour les fabriquer et ce qu'il est licite également de porter en fonction de son rang.

Par derrière cependant, c'est bien la question de la valeur qui est aussi posée. L'excès du luxe détourne le capital d'une utilisation plus conforme au bien commun, c'est-à-dire qu'il le détourne de l'investissement productif et il l'immobilise dans des objets inutiles, voire socialement dangereux. Or, l'usage chrétien des choses veut que les richesses circulent et que cette circulation serve à irriguer l'ensemble du corps social. Les classes dirigeantes, obsédées par la peur d'un appauvrissement de la société du fait d'achats trop coûteux effectués à l'étranger réagissent en fonction de cette crainte: les mesures prises contre le luxe le sont d'abord en vue de préserver les patrimoines familiaux et d'éviter leur dispersion. C'est pour conserver la richesse à l'intérieur du corps social de la ville que ces mesures sont prises.

12 G. TODESCHINI, Richesse franciscaine. De la pauvreté volontaire à la société de marché, Paris, 2008.

13 C. DE LA REONCIÈRE, Prix et salaires à Florence au XIV̌ siècle, 1289-1380, Rome, 1982.

14 M.G. MUZZARELLI, Una società nello specchio della legislazione suntuaria: il caso dell'Emilia Romagna, M.G. MUZZARELLI et A. CAMPANINI (éd.), Disciplinare il lusso, Rome, 2003, p. 17-32. 
Des amendes sont prévues contre ceux qui dépassent la mesure en matière de luxe : il est vraisemblable d'ailleurs que, au moins pour les vêtements, leur montant était incorporé au prix fait par le tailleur. $\mathrm{Au} \mathrm{XV}$ e siècle, la destination du montant des amendes prélevées à propos des objets de luxe peut être le Mont-de-Piété: ce qui est prélevé sur les riches comme un impôt forcé sur le superflu vient grossir le capital dont ont besoin les Monts-de-Piété (et parfois aussi les Hôpitaux) pour fonctionner ${ }^{15}$. Les objets de luxe taxés par l'amende permettent de subventionner les prêts consentis par les employés du Mont-de-Piété qui, précisément, reçoivent des objets pour gager les sommes reçues. Ce circuit organisé par certaines communes permet de résoudre partiellement la question du financement des Monts, toujours très difficile $^{16}$. Elle permet aussi de convertir la richesse en instrument de salut, ou du moins d'établir un processus qui permette de commencer à le faire: ce sont ici les objets qui sont mobilisés pour financer l'engagement de biens souvent modestes mais dont la mobilisation est bien souvent le seul moyen de survie des plus pauvres. La richesse, de ce fait, cesse d'être immobile et les objets de luxe ne renvoient plus à l'avaritia du groupe des dominants ${ }^{17}$. L'action répressive des pouvoirs publics permet ainsi la transformation des objets de luxe en instruments du salut de la collectivité. Ce sont les mêmes déterminants qui sont en cause au $\mathrm{IX}^{\mathrm{e}}$ siècle et au $\mathrm{XV}^{\mathrm{e}}$ siècle: comment faire en sorte que les richesses servent au salut? C'est l'immobilité des richesses qui est condamnable, davantage que leur possession. Il est cependant indispensable de disposer de réserves de valeur, quelle que soit la période considérée et celle-ci s'incarne évidemment dans les trésors, dont la possession est une nécessité.

\section{Le trésor: objets sacrés et réserve de valeur}

La question de la valeur des choses est liée à la fonction des trésors. L'objet placé dans le trésor, parce qu'il appartient à l'histoire d'un peuple ou à celle d'une famille et qu'il renferme une part du prestige de ceux-ci, ne peut évidemment aisément recevoir une valeur en équivalent monétaire. Pourtant tout le monde sait que les objets du trésor ont quelque chose de particulier et que c'est aussi parce que leur valeur est grande qu'ils ont mérité d'y être inclus ${ }^{18}$.

Encore faut-il se mettre d'accord sur ce à quoi servent les trésors. Dans le monde germanique du haut Moyen Âge, qu'il soit mérovingien, wisigoth ou lombard, le trésor est un élément de la souveraineté. Il concentre les moyens financiers du roi, son prestige, réifié littéralement à l'intérieur d'objets symbolisant le pouvoir du fait de leur fonction, de leur beauté ou tout simplement de leur histoire. Leur équivalent

15 M.G. MUZZARELLI, Il denaro e la salvezza. L'invenzione del Monte di Pietà, Bologne, 2001.

16 L. FONTAINE, L'économie morale : pauvreté, crédit et confiance dans l'Europe préindustrielle, Paris, 2008 (NRF essais).

17 Voir, sur cette notion durant le haut Moyen Âge : V. TONEATTO, op. cit., 2012.

18 S. GELICHI et C. LA ROCCA, Tesori : forme di accumulazione della ricchezza nell'alto Medioevo (secoli V-XI), Rome, 2004. 
monétaire n'est pas une question que l'on puisse aisément faire entrer en ligne de compte.

Ainsi, par exemple, en Espagne, au début du VII siècle lors de sa révolte contre Swinthila Sisenand demanda l'aide de Dagobert et des Francs; il promit de lui offrir un plateau d'or pesant 500 livres. Celui-ci aurait été donné deux siècles auparavant par Aetius au roi Thorismond. Les grands s'opposèrent à la cession de cet objet, non pas tant à cause de sa valeur mais à cause de sa signification symbolique. Datant du Ve siècle, contemporain de la fin de l'ethnogenèse des Goths, il était surtout antérieur à Vouillé: le remettre à Dagobert eût été faire un acte d'allégeance proprement insupportable. Il ne représentait pas seulement la souveraineté des Goths mais aussi leur indépendance à l'égard du pouvoir franc. En revanche, Sisenand proposa une somme d'argent extrêmement élevée, 200.000 sous selon Frédégaire, transformant ainsi ce qui aurait pu être considéré comme une marque de soumission en un acte commercial peu flatteur pour Dagobert dont l'alliance était évaluée à l'instar d'une marchandise et non pas recherchée comme une preuve de son amitié ${ }^{19}$. Le don de la table aurait pu passer pour un munus, c'est-à-dire d'un cadeau à l'intérieur d'une relation d'inférieur à supérieur à quoi aurait répondu l'aide militaire: autrement dit, on serait bel et bien entré dans une relation tributaire qui aurait signifié la soumission des Goths et non le maintien d'une relation d'égalité, ce que suppose précisément l'échange commer$\operatorname{cial}^{20}$. L'achat de l'aide militaire, même à un prix exorbitant, maintient au moins la forme de l'alliance, même si dans la réalité elle implique la soumission. Le langage du don aurait été dans ce cas celui de la soumission. Le choix de ne se séparer que des espèces monétaires indique donc un choix politique effectué sous la contrainte de l'aristocratie wisigothe qui, consciente des enjeux, n'était pas disposée à abandonner avec ses objets symboliques une partie de son indépendance. Il y aurait donc un complet contresens à voir dans les 200.000 sous le prix d'un objet. Les deux choses, la valeur de l'objet d'exception et le montant monétaire proposé à Dagobert doivent être séparées. Il s'agit ici d'un objet porteur de l'identité d'une nation, donc d'un objet qui, sous sa beauté et le luxe de sa forme, incorpore des valeurs autres que des valeurs d'usage ou d'échange: il relève en fait de la catégorie du sacré et ne saurait être dégradé du fait de son inclusion dans une transaction diplomatique. L'opération faisait sens parce que Sisenand disposait de réserves monétaires presque illimitées selon les critères du VII siècle.

Il arrive cependant que le trésor soit vide et qu'il ne soit plus possible de monnayer les alliances ou de payer les mercenaires: le recours à ceux-ci est normal dans certaines sociétés, comme la société lombarde de la seconde moitié du IX ${ }^{\mathrm{e}}$ siècle. Durant les guerres civiles des années 840-850, le prince Siconolf de Capoue recruta des mercenaires espagnols, vraisemblablement musulmans, pour combattre ses ennemis de

19 FRÉDÉGAIRE, Chronicorum quae dicuntur Fredegarii scholastici, IV, 73, MGH, SS. Rerum Merov. B. KRUSCH (éd.), pp. 157-158 ; nouvelle édition: A. Kusternig, Darmstadt, 1982 (Quellen zur Geschichte des 7. und 8. Jahrunderts). Trad. O. Devillers et J. Meyers, Turnhout, 2001.

20 J.L. NELSON, op. cit., 2010. 
Salerne ${ }^{21}$. Le Mont-Cassin s'est vu alors contraint de prêter des sommes importantes au prince et dut pour ce faire se dépouiller de son trésor au cours d'une série d'emprunts forcés qui donnèrent lieu à des tractations importantes au cours desquelles on en vint à évaluer les pièces du trésor accumulé depuis un siècle au Mont-Cassin. Les pièces d'orfèvrerie, surtout les plus anciennes, furent pour certaines pesées, d'autres furent estimées à un prix de marché sans qu'aucun compte ne soit tenu du statut des objets: certains pouvaient être des objets de culte mais cela ne ralentit en rien les opérations, leur caractère éventuellement sacré n'étant en aucune manière pris en considération par l'abbé ou par le prince. Ainsi, un objet liturgique, une nappe d'autel en soie ornée de pierres précieuses, fut par exemple estimée à 10.000 sous siciliens. Pesés ou estimés, ces objets avaient donc un prix, fort bien connu des différents acteurs ${ }^{22}$.

Le prince, qui allait devoir fondre les pièces d'orfèvrerie s'engageait par écrit à restituer non pas les objets eux-mêmes mais leur valeur. Les objets précieux, présents dans le trésor depuis un certain temps, parfois depuis l'époque de Pépin le Bref et de Carloman, ne pouvaient faire l'objet d'une estimation, du simple fait qu'ils n'avaient pas circulé depuis le temps de leur acquisition et que, même alors, leur valeur n'avait pas nécessairement été dévoilée, parce que ce n'était pas une question pertinente pour le donataire comme pour le donateur. Ne pouvant être évalués, les objets précieux étaient simplement pesés. Cela implique que, une fois emportés par le prince, ils seraient vendus au prix de l'or, sans tenir compte de l'éventuelle plus-value que leur passé prestigieux, leur ancienneté ou la qualité de leur travail pourrait leur conférer. Pour les objets donnés récemment, on connaissait parfois les prix : une couronne en or ornée d'émeraudes, qui avait été offerte par Siconolf lui-même et qui avait appartenu à son père, fut estimée à 3.000 sous.

Ce trésor, au demeurant, ne contenait pas que des objets. Le monastère détenait aussi des liquidités, pour un montant d'au moins 30.000 sous, soit 1.500 livres, en différentes monnaies. En bref, quelle qu'ait pu être sa valeur symbolique et sa fonction liturgique - il contenait évidemment nombre de vases sacrés -, il était considéré non comme un signe mais comme une richesse que le prince s'autorisait à mobiliser à son profit, mais pas en dehors de toute règle, puisque, à chaque opération (il y eu en tout 3 emprunts forcés), il établissait un scriptum redditionis, une reconnaissance de dette. De surcroît, l'opération ne fut pas nécessairement réalisée à perte. Le Mont-Cassin finit en effet par recevoir des donations de terres d'une très grande importance: tout se passe comme si l'emprunt forcé avait reçu aussi une garantie hypothécaire.

$\mathrm{Au} \mathrm{XII}$ siècle, en Castille, des procédures identiques sont imaginées par la reine Urraca pour financer la guerre civile qu'elle doit mener dans les années 1110-1120 contre Alphonse le Batailleur. Elles sont ambivalentes: la reine obtenait qu'on lui

21 Chronica monasterii casinensis, MGH, SS XXXIV, I, H. HOFFMANN (éd.), chap. 26, pp. 74-76; A. CITARELLA et H. WILLARD, The ninth-century treasure of Monte Cassino in the context of political and economic developments in South Italy, Montecassino, 1983, p. 86.

22 Sur l'importance de la pesée dans l'estimation des valeurs, et sur le caractère courant de cette opération : S.E. KRUSE, "Ingots and Weight Units in Viking Age Silver Hoards", World Archaeology, 20, 2 (1988), pp. 285-301. 
remît des trésors composés d'espèces monétaires islamiques, de lingots ou de métal précieux estimé au poids et d'objets, des coupes, des vases des pièces de vaisselle, des bijoux, des ornements sacrés comme des tables d'autel. En même temps, les concessions qu'elle était amenée à faire aux églises étaient réellement importantes, puisqu'elle cédait aussi bien des droits politiques que des terres: elle payait donc très cher, en concessions politiques et en pouvoir, l'obtention des liquidités dont elle était dépourvue. Le cadre de la négociation était dans ce cas très défavorable à la reine. Le coût des objets ainsi obtenus doit se mesurer aussi en terme de prestige et d'efficacité du pouvoir et leur valeur n'est pas seulement celle qu'en tire Uracca sur les marchés des biens précieux. Elle se trouve aussi dans l'obligation faite à la reine de renégocier les termes de son pouvoir. N'ayant pas la force de procéder à des emprunts forcés et les églises ne lui concédant aucun crédit, elle doit littéralement vendre des éléments de son pouvoir, sans être pour autant en mesure de fixer elle-même le prix ${ }^{23}$.

Nous venons d'examiner certaines des conditions de circulation des objets précieux. Leur affectation ou leur attribution, par le don ou par le legs, permet de classer les individus et les familles. La possession et l'ostentation de certains types d'objets est ainsi synonyme de statut ou indicateur de rang. Les donations d'objets auxquelles nous venons de faire allusion, en particulier celles voulues par les testaments, permettent de faire circuler les objets parés de prestige et de les répartir à l'intérieur d'un groupe restreint qui bénéficie ainsi de l'aura de ces objets et des qualités dont la possession par un homme particulièrement prestigieux les a fait bénéficier. Nous avons vu aussi que la circulation des objets de prestige était associée ou opposée à des circulations proches d'échanges commerciaux, les emprunts forcés pouvant aussi être lus comme des prêts hypothécaires (dans le cas du Mont-Cassin) ou comme des ventes forcées de droits politiques (dans le cas d'Urraca).

\section{Circulations contraintes: pillages, vols et gages}

Comment se sert-on enfin des objets de luxe? Ils ont une valeur d'usage, évidemment, et, en tant que marqueurs sociaux, ont une fonction essentielle dans les sociétés où l'ostentation des richesses est importante. Les objets de luxe se montrent donc, qu'il s'agisse de parures, de vaisselle ou de vêtements. Ils sont d'abord des figures particulières des biens d'usage courant. Mais, parce qu'ils ont une valeur monétaire importante, ils servent aussi à autre chose et, notamment de gages. On y a déjà fait allusion.

Il faut parfois, pour parvenir à survivre, se séparer provisoirement de ses objets de propriété. Fréquemment, ce sont les objets possédés par les femmes, mais pas seulement, qui font l'objet de tels transferts et, en particulier, les bijoux. Le prêt sur gage est une nécessité de la vie économique contre laquelle personne ne peut rien, malgré les tentatives de l'Église d'en interdire l'usage. Pour le petit peuple, c'est souvent une question de survie. Mais c'est aussi une façon pratique et rapide d'avoir

23 P. MARTÍNEZ SOPENA, "La circulation des objets en temps de guerre. Les années de la reine Urraca (León et Castille, vers 1110-1130)", L. FELLER et A. RODRÍGUEZ (éd.), op. cit., 2013, pp. 257-282. 
accès à un capital monétaire. À la fin du Moyen Âge comme à l'époque moderne, la plupart des objets de luxe détenus par les familles les moins à leur aise sont considérés comme des réserves de valeur ${ }^{24}$. L'une des motivations de leur acquisition est la volonté de se protéger contre les difficultés passagères ou non que peut rencontrer une famille dans le cours de son existence. La difficulté vient de deux points. Le premier est le taux d'intérêt souvent d'autant plus élevé que le prêt à court terme. Le second est l'incertitude existant sur le sort des gages. Ceux-ci sont fréquemment mis en circulation, revendus ou prêtés, avant même que le délai du prêt soit écoulé, ce qui provoque d'inévitables tensions et des conflits. Le Mont-de-Piété, entre autres avantages, garantit que le gage sera restitué si la somme prêtée est rendue. C'est donc une considérable sécurité.

N'importe quel objet peut exerce cette fonction de réserve: les objets déposés au $X V^{e}$ siècle au Mont-de-Piété sont le luxe des pauvres. C'est en tout cas leur superflu dont ils se séparent afin soit d'obtenir de quoi survivre, soit d'obtenir un capital qui permette de se lancer dans de petites entreprises. Ainsi, par exemple, les petits commerces de denrées alimentaires dépendent-ils sans doute pour leur existence de prêts à très court terme (la journée ou la semaine) afin que le commerçants puissent se procurer les denrées. Les objets de propriété non strictement indispensables ont une fonction quasi monétaire et servent à fournir les liquidités qui manquent et dont on a besoin. Engager des objets et les retirer ensuite est une possibilité normalement utilisée pour obtenir les liquidités nécessaires au commerce.

Le recours au prêt sur gage n'est pas nécessairement la voie choisie par les acteurs qui peuvent préférer des procédures plus complexes si leurs affaires l'exigent. D. Smail a montré ainsi que, en cas de faillite, il était plus avantageux, dans la Marseille du XIV siècle, d'être saisi que d'emprunter sur gage mobilier. La saisie implique un considérable déshonneur, parce qu'elle rend publique l'incompétence du failli et qu'elle est criée publiquement par la ville ${ }^{25}$. Mais elle se termine par une vente des objets: or, le prix du marché est normalement supérieur à ce que l'on peut tirer des objets engagés et il est donc plus avantageux d'être saisi que de recourir au crédit, quitte à devoir racheter les objets saisis ${ }^{26}$.

\section{Conclusion}

Les objets de luxe exercent donc des fonctions nombreuses. Marqueurs de statut, ils ont une valeur sociale de ce fait. Les mettre en circulation, les donner ou les léguer,

24 L. FONTAINE et ASSOCIATION EMMAÜS, Pauvreté et stratégies de survie : une conférence débat de l'Association Emmaüs, 21 novembre 2007, Paris, 2008, cycle «La rue?, parlons-en!».

25 Sur la criée, voir D. LETT et N. OFFENSTADT (éd.), Haro ! Noël! Oyé ! Pratiques du cri au Moyen Âge, Paris, 2003 et, en dernier lieu: P. CHASTANG, "Le gouvernement urbain, la parole et l'écrit. Autour de quatre criées urbaines montpelliéraines des années 1330”, L. GALANO et L. LAUMONIER (dir.), Montpellier au Moyen Âge (sous presse).

26 D.L. SMAIL, "Les biens comme otages. Quelques aspects du processus de recouvrement des dettes à Lucques et à Marseille à la fin du Moyen Âge”, L. FELLER et A. RODRÍGUEZ (éd.), op. cit., 2013, pp. 365-384. 
c'est faire bénéficier leurs nouveaux détenteurs d'une partie des qualités de leur précédent possesseur. Incorporant en eux des valeurs immatérielles, ils sont les vecteurs et les agents de transmission du prestige social. Il n'y a aucun intérêt à les faire circuler par le marché, justement parce qu'ils sont personnalisés et que leur valeur provient précisément du lien qu'ils entretiennent avec leurs propriétaires. Proprement immatérielle, cette valeur-là ne peut être mesurée ni transformée en équivalent monétaire.

Dès lors qu'ils sont achetés, leur détention est suspecte: les lois somptuaires semblent toutes provenir d'une même méfiance envers les nouveaux groupes sociaux émergés en ville et envers leur enrichissement. Plus profondément, la présence de ces objets permet à certaines villes d'organiser la circulation des richesses et de convertir celles-ci non pas en d'autres richesses mais en causes de salut par l'aide effective apportée aux pauvres. L'affectation des amendes aux monts de piété, dont il vaudrait la peine de voir si elle est fréquente ou non, permet d'opérer une forme de conversion des richesses équivalentes à l'aumône mais sans doute plus efficace, le mont de piété étant d'un secours plus puissant que la seule charité.

La fonction de trésor des objets de luxe montre l'instabilité de leur statut. Incorporés dans des institutions où ils sont considérés comme relevant du sacré, ils en sortent cependant avec une assez grande facilité pour soutenir des échanges politique, guerrier ou commerciaux. Les transactions qui entourent ainsi les grands trésors du haut moyen âge montrent toute la complexité de ces objets.

Enfin, la question du gage est évidemment d'une importance extrême. L'objet engagé n'est pas perdu pour son propriétaire et peut lui revenir. Le fait que n'importe quel objet puisse être transformé en gage, y compris au cours de procédures judiciaires, montre l'importance que la question de la détention d'objets de luxe ou d'objets de prestige a dans les sociétés anciennes.

Le luxe, dans ces conditions, ne reçoit pas une définition absolue: les objets de luxe, pour toute une catégorie de la population, c'est simplement le superflu, ce qui n'est pas indispensable à la survie immédiate et qui permet simplement à la fois d'accéder au capital nécessaire à l'entreprise d'activités économiques, le plus souvent commerciales, et, le cas échéant, d'obtenir les ressources nécessaires à la survie. 